\title{
DIABETES AND PERIODONTAL DISEASE: A TWO WAY RELATION - A COMMENTARY
}

G. Jacob. Diabetes And Periodontal Disease: A Two Way Relation - A Commentary. Annal Dent Univ Malaya 2013; 20(1): $27-30$

\begin{abstract}
An association between diabetes and periodontitis has been long suspected and has now been reasonably well documented for both type I insulin dependent diabetes mellitus and type II non insulin dependent diabetes mellitus. Diabetes mellitus is a systemic complication affecting both the quality and length of life. The interrelationship between periodontitis and diabetes provides an example of systemic disease predisposing to oral infection and once the infection is established, the oral infection can exacerbate the systemic disease. Diabetes induced changes in immune functions which provide an inflammatory cell phenotype. This predisposes to chronic inflammation, progressive tissue break down and diminished tissue repair capacity. Periodontal tissues frequently manifest these changes because they are constantly wounded by substances emanating from bacterial bio films. The well informed dentist has the opportunity to be at the forefront of diagnosing diabetes. Studies have shown that nonsurgical periodontal treatment is associated with improved glycemic control in type II diabetes mellitus patient. Periodontal treatment should be undertaken along with standard measures for diabetic control and care. Prevention and control of periodontal disease must be considered an integral part of diabetes mellitus.
\end{abstract}

Keywords

Diabetes mellitus, periodontitis, non surgical periodontal therapy, glycemic control.

\section{INTRODUCTION}

Diabetes mellitus and chronic periodontitis are common chronic diseases affecting adults in the worldwide population. Diabetes mellitus is a complex disease with both metabolic and vascular components characterized by hyperglycemia due to defects in insulin secretion, insulin action or both. Patients suffering from diabetes mellitus are known to have increased susceptibility to certain infections. The two-way inter relationship between diabetes and periodontitis has been proven, and different modalities of treatment involving diabetes and periodontitis are being looked upon
Review Article

\begin{tabular}{l}
${ }^{1}$ G. Jacob \\
IDepartment of Periodontics \\
Pushpagiri College of Dental Sciences \\
Thiruvalla, Kerala, \\
India. \\
Corresponding Author: \\
Dr. Jacob George \\
Email: jacobkallarackal@gmail.com \\
\hline
\end{tabular}

\section{PREVALENCE AND INCIDENCE}

About 371 million people in the age group of 20-79 years worldwide are estimated to have diabetes in the year 2013. By 2025, the adult diabetic population is expected to double in India to about 73 million and in China to 46 million. At the same time diabetes prevalence rate is expected to increase to $2.8 \%$ of the adult population in Africa and $7.2 \%$ in South and Central America. The European and Western Pacific region with 48 and 43 million people respectively are currently having the highest number of people with diabetes (1).

In the present scenario and changing life styles, Asia will have the largest number of diabetics. Diabetes is expanding in pandemic proportions worldwide. Escalation in prevalence of diabetes appears to be more pronounced in developing countries (2). Ramachandran et al (2003) had shown that type II diabetes in children which were earlier reported mostly in developed countries such as United Kingdom, United States and Japan now occur even in developing countries (3). Obesity, parental history of type II diabetes and pubertal age appeared to be strongly associated with the disease in Asian children.

\section{DIABETES MELLITUS AND PERIODONTITIS}

An association between diabetes and periodontitis has been long suspected and has now been reasonably well documented for both type I insulin-dependent diabetes mellitus and type II non insulin-dependent diabetes mellitus. 
Shlossman et al (1990) had shown a relationship between type II diabetes mellitus and periodontal disease (4). Subjects with diabetes were shown to have a higher prevalence of periodontal disease indicating that diabetes may be a risk factor for periodontal disease. Thorstensson et al (1993) had shown that diabetics aged between 40-49 years had more periodontal pockets which were greater than or equal to $6 \mathrm{~mm}$ and more extensive alveolar bone loss than non-diabetics of the same group (5). George et al (1998) had shown that type II diabetes significantly increased the rate of alveolar bone loss progression over a 2 year period when compared to non-diabetic subjects (6). The risk for bone loss was 4.2 times greater in diabetic subjects with the greatest increase in risk occurring in patients under the age of 34 years.

\section{MECHANISM OF INTERACTION BETWEEN DIABETES MELLITUS AND PERIODONTIUM}

\section{Alterations in subgingival microbiota and gingival crevicular fluid}

Mashimo et al (1983) reported that there was an increase in Capnocytophaga species and anaerobic vibrios in diabetic patients (7). Sastrowijotosh et al (1989) showed that Capnocytophaga species were isolated at very low levels, in both the diseased and healthy periodontal pockets (8). Thus the role of Capnocytophaga species in the pathogenesis of infectious periodontal disease in patients with type I diabetes may be over estimated. The presence of Actinobacillus actinomycetem comitans and black pigmented bacteroides species were found in the periodontal microflora of the diseased pockets of the diabetic group. Zambon et al (1998) showed that sub gingival microflora in severe periodontitis patients with non insulin-dependent diabetes mellitus is similar to that found in non-diabetic adults with severe periodontitis (9). Both groups exhibited high prevalence and large proportions of gram negative anaerobes including the black pigmented bacteroides species, Porphyromonas gingivalis, Prevotella intermedia and Wolinella recta. Most of the studies showed very few differences in the sub gingival micro biota of periodontitis sites in diabetes mellitus subjects compared to similar sites with periodontitis in non diabetes mellitus subjects.

Nishimura et al (1996) had shown decreased chemotaxis of periodontal ligament fibroblasts in response to platelet derived growth factor when cultured in a hyperglycemic environment compared to normoglycemic conditions (10). Elevated glucose levels in the gingival crevicular fluid of individuals with diabetes may thus adversely affect periodontal wound healing and the local host response to microbial challenge.

\section{Collagen metabolism, advanced glycation end products} and wound healing

Changes in collagen synthesis, maturation and turn over are common in diabetes mellitus. Since the periodontium is composed primarily of collagen, these changes in collagen metabolism may contribute to alterations in wound healing and to periodontal disease initiation and progression. In addition to decreased synthesis newly formed collagen is susceptible to degradation by collagenase, a matrix metalloproteinase which is elevated in diabetic patients including the periodontium. A greater percentage of this collagenase is in the active form in patients with diabetes mellitus compared to non-diabetics (11). In addition to the decreased collagen production and increased collagenase activity, collagen metabolism is altered by accumulation of advanced glycation end products in the periodontium. Changes affecting the blood vessels of the glomerulus and retina can also occur in the periodontium. Increased thickness of gingival capillary endothelial basement membrane and walls of small blood vessels may be seen in diabetic individuals (12). The thickening may impair the exchange of oxygen and metabolic waste products across the basement membrane. There was a demonstrable two-fold increase in accumulation of advanced glycation end products (AGE's) in the periodontium of diabetes patients compared to non-diabetic individuals (13). Increased collagenase activity in diabetes mellitus results in greater degradation of newly formed more soluble collagen. Conversely, the accumulation of AGE's causes greater cross linking of mature collagen. The net effect is a predominance of older highly cross linked AGE's modified collagen. In the capillaries, this accumulation of highly cross linked collagen in the basement membrane increases membrane thickness. These events play a role in altering the tissue response to periodontal pathogens resulting in increased severity and progression of periodontitis (14).

\section{Changes in host immunoinflammatory response}

With few major differences in the sub gingival microbiota between diabetic and non-diabetc patients, attention is now turned to the differences in the host immunoinflammatory response. Polymorphonuclear leukocyte plays a major role in maintaining a healthy periodontium in the face of periodontal microorganisms. In diabetes mellitus, there is a reduction in polymorphonuclear leucocyte function including chemotaxis, adherence and phagocytosis. Diabetes mellitus patients with severe periodontitis have been shown to have reduced polymorphonuclear leucocyte chemotaxis compared to diabetic patients with mild to moderate periodontitis. Defects affecting polymorphonuclear leucocytes, the first line of defense against subgingival microbial agents, may result in significantly increased tissue destruction.

In addition to the polymorphonuclear leucocytes anothercritical cell in the periodontal immunoinflammatory 
response to pathogen is the monocyte / macrophage lineage. Diabetic patients possess a hyper-responsive monocytes / macrophages in which stimulation by bacterial antigen results in stimulated production of proinflammatory cytokines such as tumour necrosis factor - a by monocytes in diabetic patients. The production of prostaglandin $\mathrm{E}_{2}$ and interleukin $1 \mathrm{~b}$ were also significantly higher than in non-diabetics with a similar degree of periodontal destruction. Advanced glycation end product formation plays an important role in up regulation of monocyte/macrophage lineage. Accumulation of AGE's in the periodontium stimulates migration of monocytes to the site. Once in the tissue, AGE's interact with the receptor of AGEs (RAGE) on the cell surfaces of the monocytes at local site. The AGE-RAGE interaction then induces a change in the monocyte phenotype which significantly increases pro-inflammatory cytokine production. This explains increased gingival crevicular fluid production of tumor necrosis factor- $\propto$, prostaglandin $\mathrm{E}_{2}$ and interleukin$1 \beta$ seen in diabetic patients with periodontitis (15).

\section{PERIODONTAL THERAPY ALTERS INSULIN REQUIREMENT}

Some association exists between periodontal healing response and improved metabolic control of blood glucose levels. Metabolic control of blood glucose level can be altered by controlling periodontal inflammation (16). Patients treated with ultrasonic scaling and curettage combined with anti-microbial regimen showed improvement in clinical parameter and microbial reduction. Effective treatment of periodontal infection and reduction of periodontal inflammation is associated with a reduction in the levels of glycosylated hemoglobin. Control of periodontal infections should thus be an important part of the overall management of diabetic patients (17). Chronic infections such as periodontal disease have a negative effect on the metabolic control in diabetics. Therefore elimination of such infection and associated inflammation, and pharmacologic intervention to inhibit glycosylation, results in short term improvement in the $\mathrm{HbA}_{\mathrm{c}}$ concentration. The treatment approach included both antimicrobial agents and pharmacological modulation of host response. Hence the elimination of periodontal inflammation resulted in a significant reduction in the concentration of glycosylated heamoglobin (18).

A study was conducted by Karagalaineh et al (1997) to explore the effect of periodontal therapy on glycemic control in patients with type 2 diabetes mellitus (19). During the nine month observation period there was a $6.7 \%$ improvement in glycemic control in the control group when compared to a $17.1 \%$ improvement in the treatment group. There were studies conducted against the proposed hypothesis that periodontal treatment improves glycemic control as well (20).

\section{CONCLUSION}

Ensuring oral health in patients with diabetes requires an expanded scope of medical and dental knowledge. There is undoubtedly a close relationship between diabetes and periodontitis. Diabetes increases the risk of periodontal destruction especially in patients whose glycemic control is poor. These patients are most likely to report to the dental office with significant periodontal treatment needs. All diabetic patients should have routine dental evaluation and preventive therapy.

\section{REFERENCES}

1. Sicree R, Shaw J and Zimmet P. The global burden of diabetes, Diabetes and impaired glucose tolerance: preva-lence and projections. In: Gan D eds. Diabetes Atlas. 2nd ed. International Diabetes Federation, Brussels 2003; pp 15-71.

2. Mohan.V. Why are Indian diabetic subjects more prone to coronary artery disease. International Journal of Diabetes in Developing Countries 2004; 24 (1): 1-6.

3. Ramachandran A, Snehalatha C, Satyavani K, Sivasankari S and Vijay V. Type 2 diabetes in Asian Indian Urban children. Diabetes Care 2003; 26 (4): 1022-1025.

4. Shlossman, M, Knowler WC, Pettit DJ and Genco RJ. Type II Diabetes mellitus and periodontal disease. J Am Dent Association 1990; 121 (4): 5326.

5. Thorstensson $\mathrm{H}$ and Hugosson A. Periodontal disease experience in adult long duration insulin dependent diabetics. J Clin Periodontol 1993; 20 (5): 352-358.

6. Taylor GW, Burt BA, Becker MP, Genco RJ, Schlossman M, Knowler WC and Pettit DJ. Insulin Dependent Diabetes Mellitus and Alveolar Bone Loss Progression over 2 years. J Periodontol 1998; 69 (1): 76-83.

7. Mashimo PA, Yamamoto Y, Slots J, Park BH and Genco RJ. The Periodontal microflora of juvenile diabetes culture immunofluorescence and serum antibody studies. J Periodontol 1983; 54 (7): 420430. 
8. Sastrowijotosh H, Hillemans $\mathrm{P}$ and Steenberg V. Periodontal condition and microbiology of healthy and diseased periodontal pockets in Type 1 diabetes. J Clin Periodontol 1989; 16(3): 16-322.

9. Zambon JJ, Reynolds H, Fisher JG, Shlossman M, Genco RJ and Dunford R. Microbiological and immunological studies of adult periodontitis in patients with non insulin dependent diabetes mellitus. J Periodontol 1998; 59 (1): 23-31.

10. Nishimura F, Terranova VP, Foo H, Kurihara M and Murayama Y. Glucose mediated alteration of cellular function in human periodontal ligament cells. J Dent Res 1996; 75 (9): 1664-1671.

11. Sorsa $T$, Ingman $T$, Suomalomen $K$, Halinen $S$, Saari H, Konttinen YT, Vitto VJ and Gollub L.H. Cellular source and tetracycline inhibition of gingival crevicular fluid collagenase of patients with diabetes mellitus. J Clin Periodontol 1992; (19): 146-149.

12. Schmidt AM, Weidman E, Yan SD, Hori O, Cao $\mathrm{R}$, Brett JG and Lamster IB. Advanced glycation endproducts induce oxidative stress in the gingiva - A potential mechanism underlying accelerated periodontal disease associated with diabetes. J Periodontal Res 1996; (7): 508-515.

13. Seppala B, Sorsa $\mathrm{T}$ and Ainamo J. Morphometric analysis of cellular and vascular changes in gingival connective tissue in long term insulin dependent diabetes. J Periodontol 1997; 68 (12): 1231-1245.
14. Mealy BL and Mortiz JL. Hormonal influences and effects of diabetes mellitus and endogenous female sex steroid hormones on the periodontium. Periodontol 2003; 32 (1): 59-81.

15. GIiovanni E, Salvi, Behnazyalda, Collins JG. Inflammatory mediator response as a potential risk factor for periodontal disease in insulin dependent diabetes mellitus patients. J Periodontol 1997; 68 (2): $127-135$.

16. Lacopino AM. Periodntitis and diabetes interrelationships. Role of inflammation. Ann of Periodontol 2000; 6 (1): 125- 37.

17. Miller LS, Agnes M, Newbold D and Mary E. The relationship between reduction in periodontal inflammation and diabetic control (A report of 9 cases). J Periodontol 1992; (63): 843-848.

18. Grossi GS, Skrepcinski FB, Thomas P and Alex DCR. W.H.O. Treatment of periodontal disease in diabetics reduces glycated heamoglobin. J Periodontol 1997; 68 (8): 713-719.

19. Karagalaineh TK. Periodontal disease related to diabetic status - A pilot study of the response to periodontal therapy in type I diabetics. J Clin Periodontol 1997; 24 505-510.

20. Aldridge JP, Lester V, Watts TLP and Collins A. Single blind studies on the effect of improved periodontal health on metabolic control in type I Diabetes Mellitus. J.Clin Periodontol 1995; 22 (4): 217-275. 